Received 18 April; accepted 28 May 1997

Broadie, K. et al. Syntaxin and synaptobrevin function downstream of vesicle docking in Drosophila. Neuron 15, 663-673 (1995).

2. Pieribone, V. A. et al. Distinct pools of synaptic vesicles in transmitter release. Nature 375, 493-497 (1995).

3. Ceccarelli, B. \& Hurlbut, W. P. Vesicle hypothesis of the release of quanta of acetylcholine. Physiol. Rev 60, 396-441 (1980).

4. Hunt, J. M. et al. A post-docking role for synaptobrevin in synaptic vesicle fusion. Neuron 12, 1269 1279 (1994).

Gillis, K. D. \& Chow, R. H. Kinetics of exocytosis in adrenal chromaffin cells. Sem. Cell Dev. Biol. (in the press).

6. Trifaro, J. M. \& Vitale, M. L. Cytoskeleton dynamics during neurotransmitter release. Trends Neurosci. 16, 466-472 (1993)

7. Stout, A. L. \& Axelrod, D. Evanescent field excitation of fluorescence by epillumination microscopy. Appl. Optics 28, 5237-5242 (1989).

8. Moser, T. \& Neher, E. Rapid exocytosis in single chromaffin cells recorded from mouse adrenal slices. $J$. Neurosci. 17, 2314-2323 (1997).

9. Stevens, C. F. \& Tsujimoto, T. Estimates for the pool size of releasable quanta at a single central synapse and for the time required to refill the pool. Proc. Natl Acad. Sci. USA 92, 846-849 (1995).

10. von Gersdorff, H. \& Matthews, G. Depletion and replenishment of vesicle pools at a ribbon-type synaptic terminal. J. Neurosci. 17, 1919-1927 (1997).

11. Schroeder, T. J., Jankowski, J. A., Senyshyn, J., Holz, R. W. \& Wightman, R. M. Zones of exocytotic release on bovine adrenal medullary cells in culture. J. Biol. Chem. 269, 17215-17220 (1994).

12. Ryan, T. A. \& Smith, S. J. Vesicle pool mobilization during action potential firing at hippocampa synapses. Neuron 14, 983-989 (1995).

13. Betz, W. J. \& Wu, L.-G. Kinetics of synaptic-vesicle recycling. Curr. Biol. 5, 1098-1101 (1995).

14. Evans, L. L. \& Bridgman, P. C. Particles move along actin filament bundles in nerve growth cones. Proc. Natl Acad. Sci. USA 92, 10954-10958 (1995).

15. Lee, R. W. \& Trifaro, J. M. Characterization of anti-actin antibodies and their use in immunocytochemical studies on the localization of actin in adrenal chromaffin cells in culture. Neurosci. 6, 20872108 (1981)

16. Cheek, T. R. \& Burgoyne, R. D. Nicotine-evoked disassembly of cortical actin filaments in adrenal chromaffin cells. FEBS Lett. 207, 110-114 (1986).

17. Parsons, T. D., Coorssen, J. R., Horstmann, H. \& Almers, W. Docked granules, the exocytic burst, and the need for ATP hydrolysis in endocrine cells. Neuron 15, 1085-1096 (1995).

18. Tse, F. W., Tse, A., Hille, B., Horstmann, H. \& Almers, W. Local $\mathrm{Ca}^{2+}$ release from internal stores controls exocytosis in pituitary gonadotrophs. Neuron 18, 121-132 (1997).

19. Weibel, E. R., Gonzague, S. K. \& Scherle, W. F. Practical stereological methods for morphometric cytology. J. Cell Biol. 30, 23-38 (1966)

20. Gingell, T. \& Todd, I. Interference reflection microscopy. A quantitative theory for image interpretation and its application to cell-substratum separation measurement. Biophys. J. 26, 507-526 (1979).

21. Chow, R. H. \& von Rüden, L. in Single-Channel Recording (eds Sakmann, B. \& Neher, E.) 245-275 (1995)

22. Ghosh, R. N. \& Webb, W. W. Automated detection and tracking of individual and clustered cell surface low density lipoprotein receptor molecules. Biophys. J. 66, 1301-1318 (1994).

23. Wightman, R. M. et al. Temporally resolved catecholamine spikes correspond to single vesicle release from individual chromaffin cells. Proc. Natl Acad. Sci. USA 88, 10754-10758 (1991).

24. Terakawa, S., Fan, J. H., Kumakura, K. \& Ohara-Imaizumi, M. Quantitative analysis of exocytosi directly visualized in living chromaffine cells. Neurosci. Lett. 123, 82-86 (1991)

Acknowledgements. We thank J. Howard, M. Lindau, W. M. Roberts and T. Soldati for their helpful suggestions on the manuscript.

Correspondence and requests for materials should be addressed to W.A. (e-mail: almers@mzf.mpimfheidelberg.mpg.de)

\section{Optical detection of a quantal presynaptic membrane turnover}

\section{Timothy A. Ryan $\star$, Harald Reuter $\dagger$ \& Stephen J Smith}

Department of Molecular and Cellular Physiology, Stanford University Medical School, Stanford, California 94305, USA

$\dagger$ Department of Pharmacology, University of Bern, 3010 Bern, Switzerland

Exploration of the mechanisms and plasticity of synaptic transmission has been hindered by the lack of a method to measure single vesicle turnover directly in individual presynaptic boutons at isolated nerve terminals. Although postsynaptic electrical recordings have provided a wealth of invaluable basic information about quantal presynaptic processes ${ }^{1}$, this approach has often proved difficult to apply at most central nervous system synapses $^{2-6}$. Here we describe the direct optical detection of single quantal events in individual presynaptic boutons of cultured hippocampal neurons. Using the fluorescent dye FM 1-43 as a tracer for presynaptic endocytosis ${ }^{7-10}$, we have characterized

Present address: Department of Biochemistry, Cornell University Medical College, 1300 York Avenue New York, New York 10021, USA. both evoked and spontaneous components of presynaptic function at the level of individual quanta. Our results are consistent with quantal interpretations of previous electrophysiological analyses $^{1-6}$ and provide new information about the unitary membrane recycling event and its coupling to individual action potential stimuli, about spontaneous vesicle turnover at individual boutons, and about the numbers of vesicles recycling at individual boutons.

The strategy we used for optical detection of single presynaptic quanta builds upon earlier studies using the fluorescent dye FM $1-43$ as a marker for synaptic vesicle turnover ${ }^{7-10}$. So far there has been no direct evidence demonstrating that uptake and release of such a vesicle market can occur in quantized units corresponding to single vesicles. Our approach has been to include refinements designed for the measurement of very weak presynaptic fluorescence signals. Single-molecule fluorescence detection ${ }^{11-14}$ has shown that detection of weak signals is limited primarily by the presence of background fluorescence in instruments and specimens. We used confocal laser scanning microscopy to minimize instrumental autofluorescence background, and sparse monolayer cell cultures and the briefest possible staining exposures to FM 1-43 to minimize specimen fluorescence backgrounds.

A scheme of our experimental protocol is shown at the top of Fig. 1. Figure 1a is a Nomarski image illustrating the site of a putative axo-dendritic synaptic junction (arrow). Figure 1b, c shows fluorescence images of the same field, acquired sequentially after FM 1-43 loading with a single action potential (AP) stimulus (image A in the schematic): both panels show a small area of bright fluorescence localized to the junction site-measurement reproducibility is excellent and is generally sustained for hours at unstimulated boutons. Figure 1d, e shows reduction in fluorescence produced by a long unloading train of APs (image B). The fluorescence signal $\Delta F$ is defined as the difference in bouton fluorescence measurements before and after unloading (that is, images $\mathrm{A}-\mathrm{B}$ ). This $\Delta F$ signal presumably represents the amount of dye taken up into a releasable vesicular pool during the specified load. A second series (Fig. 1f-h) with more stimulation during loading (20 AP) verifies the presence of a viable presynaptic bouton at the site of interest. The 20 AP load resulted in 16-fold greater uptake of releasable dye (Fig. 1i).

To test for the postulated quantization of FM 1-43 signals, we measured fluorescence values across a large number of minimally stimulated individual boutons. Figure $2 \mathrm{a}$ represents individual measurements from a representative experiment in which just 1 AP was stimulated: each vertical set of points represents three separate measurements from the same bouton (fluorescence after loading but before unloading, black circles; the residual nonreleasable fluorescence measured after extensive unloading, white circles; and the intrinsic fluorescence of the synapse measured before loading $\left(F_{\mathrm{I}}\right)$, squares); solid, vertical lines represent $\Delta F$, and dotted lines represent amplitudes of the residual fluorescence signal $F_{\mathrm{R}}$. These data show that releasable fluorescence $(\Delta F)$ is $\sim 10$ times greater than $F_{\mathrm{I}}$, but that $F_{\mathrm{R}}$ magnitude is in the same range as the minimal $\Delta F$ signals. Because $F_{\mathrm{R}}$ became visible after even the briefest dye exposures, regardless of whether any APs are fired, this weak, non-releasable fluorescence probably reflects a residual membrane staining with no special relationship to synaptic vesicle recycling. This interpretation of $F_{\mathrm{R}}$ is further supported by the lack of any significant bouton-by-bouton correlation between $F_{\mathrm{R}}$ and $\Delta F$ values (data not shown).

Figure $2 \mathrm{~b}$ shows a histogram of the same $\Delta F$ values as in Fig. 2a. This histogram shows a distinct peak at a $\Delta F$ amplitude of 63 photoelectrons. There are also hints of secondary and tertiary peaks at double and triple that amplitude, but clearly the number of observations is inadequate for much certainty here. By pooling observations from several experiments, however, a multimodal response amplitude distribution becomes much more obvious. Figure 3a shows a frequency histogram of the different values of 
$\Delta F$ measured from 409 individual boutons stimulated by 1, 2 or 3 APs. (The 2- and 3-AP stimulus runs were included to favour the possible occurrence of more multiple-quantum events.) The histogram shows a prominent peak centred at 60 photoelectrons, with higher-order peaks appearing precisely at integral multiples. This clearly multimodal response distribution is exactly as would be predicted from the hypothesis that releasable staining is taken up in quantal units corresponding to individual synaptic vesicles.

The solid line in Fig. 3a is a curve generated as the sum of four gaussian functions centred about exact integer multiples of the mean of the first peak (see Methods). The variance within individual peaks appears to be accounted for entirely by the expected photon shot-noise statistics associated with each measurement (see Methods). The peak spacing, $F_{\mathrm{Q}}$, used to fit this histogram provides a factor that can now be used to calibrate any $\Delta F$ measurement in terms of an actual number of presynaptic quanta recycled $\left(N_{\mathrm{Q}}=\Delta F / F_{\mathrm{Q}}\right)$. This calibration also indicates that the releasable fluorescence illustrated in Fig. 2b-e corresponds to a single quantum.

Our results may underestimate the numbers of quanta turned over by minimal AP stimuli. Dye exposures were limited here to $\sim 7 \mathrm{~s}$ to minimize fluorescence backgrounds, whereas earlier work has shown that substantial AP-evoked endocytosis may trail stimulation with a half-time of as long as $20 \mathrm{~s}$. Thus, this low-noise procedure may fail to label some number of late-endocytosing vesicles ${ }^{15}$.
It has long been known ${ }^{1}$ that presynaptic quantal release can occur in a spontaneous as well as an evoked fashion. To characterize spontaneous vesicle turnover under our experimental conditions, we carried out experiments in which no APs were stimulated and in which calcium influx during any possible spontaneous APs (already minimized, if not eliminated by extracellular CNQX) were blocked by adding $100 \mu \mathrm{M}$ extracellular $\mathrm{Cd}^{2+}$. A dye exposure of $20 \mathrm{~s}$ gave the results shown in Fig. $3 \mathrm{~b}$, in which the frequency histogram of $\Delta F$ was measured at 212 different boutons from 5 different experiments. The distribution of $\Delta F$ values shows a prominent peak at 63 photoelectrons, and a second peak at twice that value. The positions of these peaks are similar to those obtained with AP stimulation (Fig. 3a). These data thus indicate that the endocytic uptake associated with spontaneous vesicular release and recycling is identical to that evoked by AP firing. These measurements also provide a means to assess frequencies of spontaneous release at individual boutons. Spontaneous recycling frequencies $\left(f_{\mathrm{s}}\right)$ were determined by measuring $N_{\mathrm{Q}}$ for a fixed dye exposure period, $\tau$, from a population of identified presynaptic boutons. The frequency $f_{s}$, calculated for each bouton as $N_{\mathrm{Q}} / \tau$, varied considerably across boutons as well as different experiments. Average values of $f_{s}\left(\left\langle f_{s}\right\rangle\right)$ determined from individual experiments spanned a tenfold range $\left(0.01 \mathrm{~s}^{-1}-0.1 \mathrm{~s}^{-1}\right)$.

This low frequency of spontaneous release indicates that spontaneous vesicle recycling must make only a very minor contribution
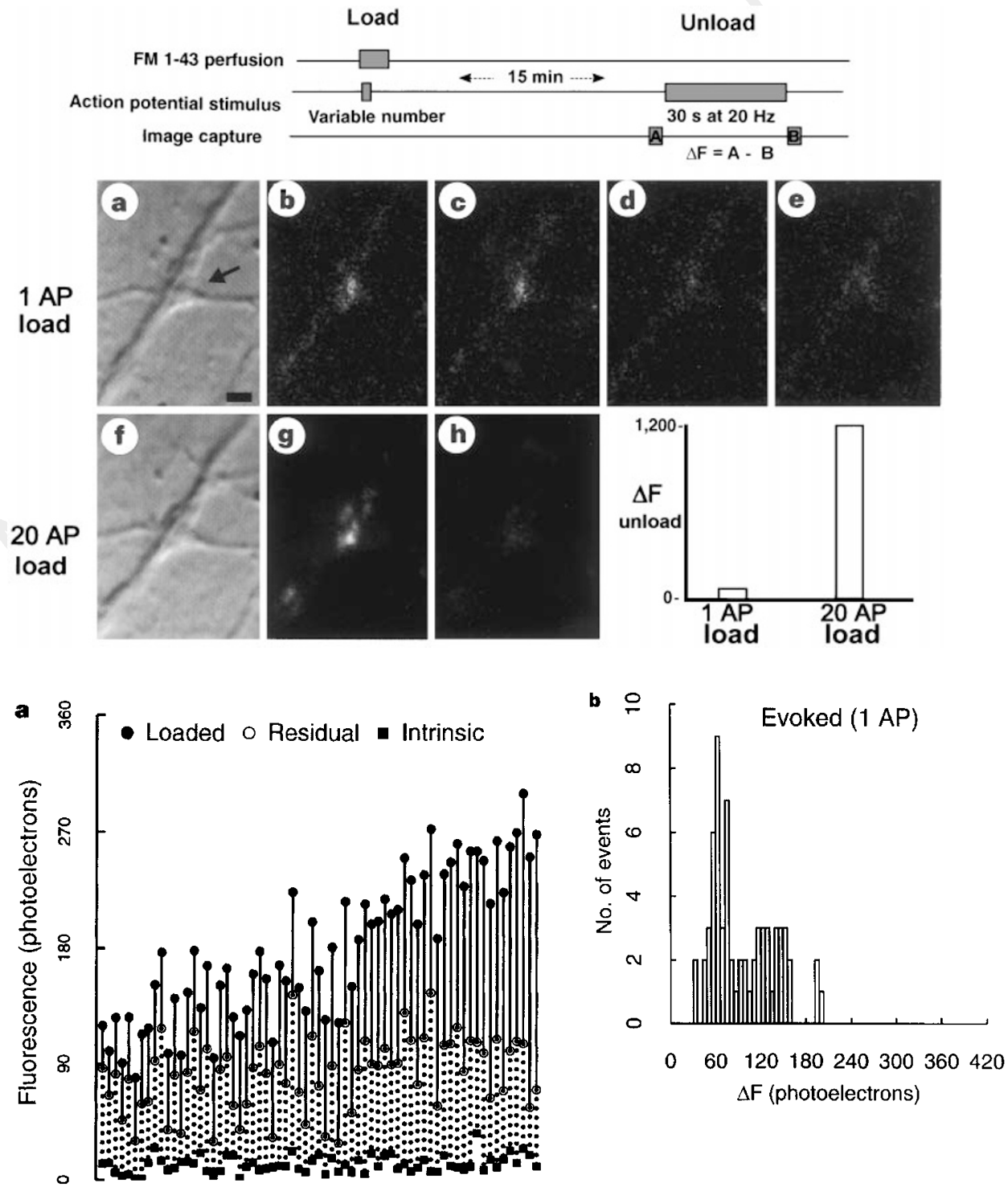

Figure 2 Fluorescence with a 1 AP loading stimulus, as measured from 67 individual boutons in a single experiment. a, Three measurements were made from each bouton: the intrinsic fluorescence before any dye exposure, $F_{\text {I }}$ (filled squares); fluorescence after loading, filled circles; fluorescence after unloading, $F_{\mathrm{R}}$ (open circles). Length of solid vertical line corresponds to releasable fluorescence $\Delta F$; length of the dotted line to the dye-related residual fluorescence. b, A frequency histogram of the same $\Delta \mathcal{F}$ data as in $\mathbf{a}$ 
to our measurement of evoked quantal activity. Figure $3 \mathrm{c}$ confirms this point: the number of quanta loaded at individual boutons by sequential stimulus episodes consisting of one and then five APs is in approximate proportion to the number of APs fired. If spontaneous quantal activity was important in dye uptake under these conditions, a disproportionately large signal would be expected from the single AP stimulus.

As a further test of the key expectation that $F_{\mathrm{Q}}$ should reflect the nature of the quantal event independently of its probability of occurrence, we reduced the probability of evoked release by using a low-calcium $(0.5 \mathrm{mM})$ high-magnesium $(3.5 \mathrm{mM})$ superfusate $^{1}$. Figure $3 \mathrm{~d}$ shows that the resulting frequency histogram is still multimodal and that the quantal spacing $F_{\mathrm{Q}}$ is essentially unchanged from that observed at normal calcium/magnesium concentrations ( $2 \mathrm{mM}$ each), even though the probability of release per AP was considerably reduced.

Estimates of the number of quanta composing the total recycling vesicle pool, $N_{\text {total }}$, were obtained by measuring the ratio $\Delta F_{\mathrm{S}} / F_{\mathrm{Q}}$ at individual boutons, where $\Delta F_{\mathrm{S}}$ is the signal obtained for saturating load conditions (400 AP load). Figure 4a shows a representative frequency distribution of different $N_{\text {total }}$ measured at 215 boutons from three experiments and indicates that $\left\langle N_{\text {total }}\right\rangle=127$. The fact that this number agrees closely with ultrastructural estimates of hippocampal vesicle pool sizes ${ }^{16,17}$ provides additional support for our conclusion that presynaptic $\Delta F$ quanta correspond to single presynaptic vesicles.

Measurements of quantal turnover per AP for brief-stimulus trains (7 AP), as well as $N_{\text {total }}$ at the same synapses, were also made. For these experiments, longer dye exposures were used (Fig. 4) to minimize the possible underestimation of quantal turnover number, at the cost of modest but tolerable increases in background fluorescence noise. Data from such experiments indicate that the average fractional turnover of the entire pool per AP obtained in a single experiment was $0.47 \pm 0.03 \%(n=54)$, in close agreement with previous estimates obtained using macroscopic release assays of FM-1-43-labelled vesicles at hippocampal boutons ${ }^{18}$. A comparison of the quantal turnover to $N_{\text {total }}$ at each synapse in a given experiment reveals a strong correlation between these two quantities (Fig. 4b). These results suggest that a single AP results in the uptake of between 0 and 3 quanta per bouton, with the average turnover across all competent boutons being $0.80 \pm 0.08$ quanta per AP. (Because of the possibility that turnover is still underestimated
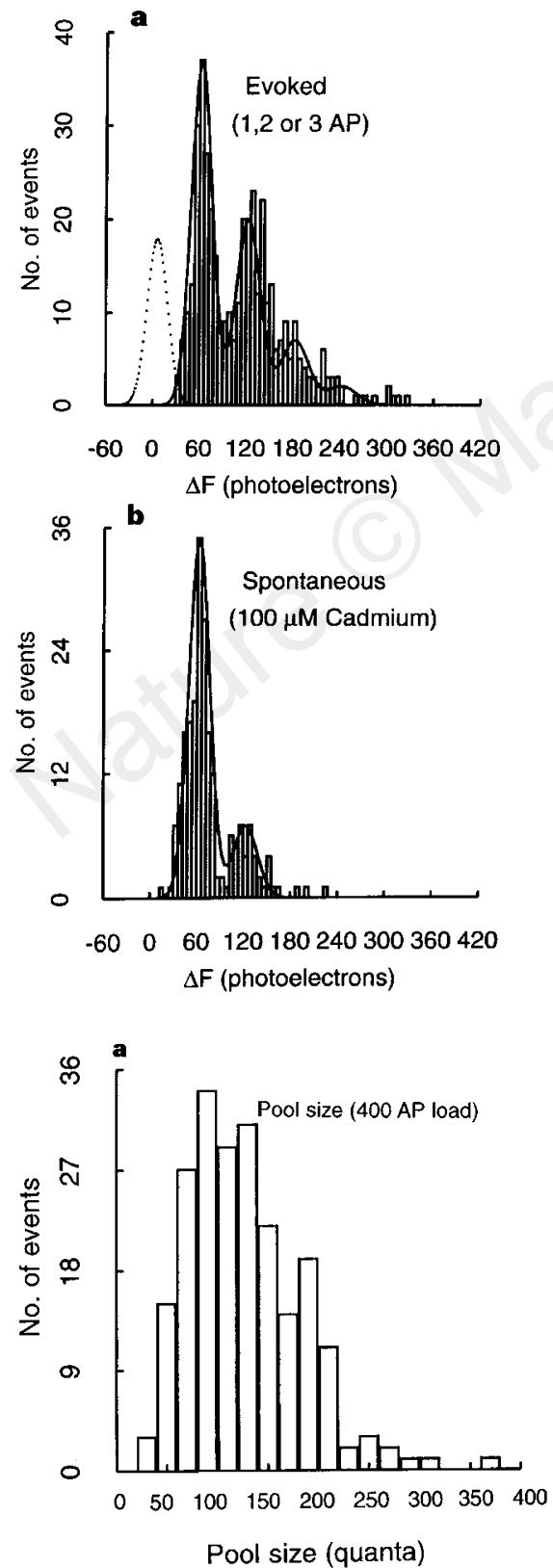
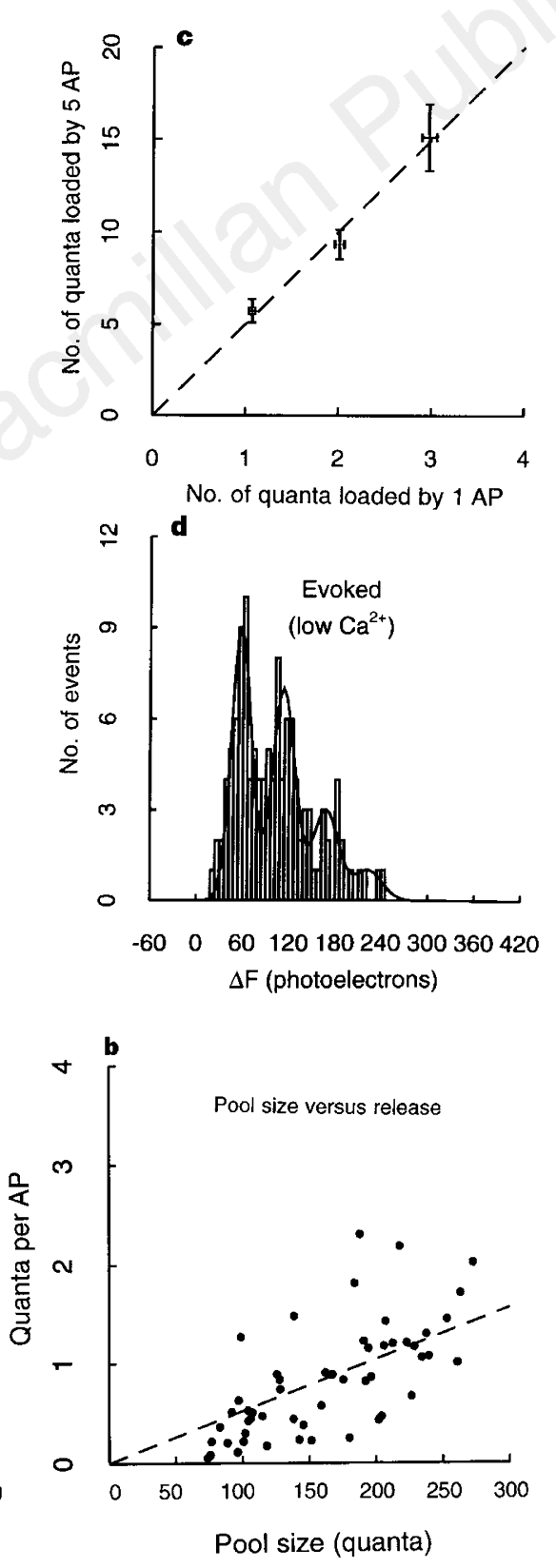

Figure 3 Minimally stimulated fluorescence signal amplitudes. a, Histogram of $\Delta F$ values from 8 experiments $(1,2$ or 3 AP load at $0.3 \mathrm{~Hz})$. Solid line is a multiple gaussian with peaks at equal 60-photoelectron intervals and widths based on a photodetection noise model (see Methods). Dotted line is a single gaussian fitted to the background noise distribution (see Methods). b. Histogram of $\Delta F$ values from spontaneous vesicle turnover (212 boutons, 5 experiments). Solid-line fit from equation (1) $\left(\alpha_{3}, \alpha_{4}\right.$, both $0 ; \mu=63$ photoelectrons). c, Scaling of quantal turnover with AP number. Sequential measurements of $\Delta F$ from 1 AP and 5 AP loads of the same bouton were binned by nearest integral value of $N_{\mathrm{Q}}$ for $1 \mathrm{AP}$ load $\left(n_{1}=44\right.$, $n_{2}=28, n_{3}=7$, errors, s.e.m.). Dotted line has a slope of 5. d, Histogram for uptake evoked by $4,5,6$ or 7 AP loads in low-Ca ${ }^{2+}$, high-Mg ${ }^{2+}$ saline (119 synapses, 4 experiments). Solid line from equation (1), with $\mu=58$ photoelectrons.

Figure 4 Estimates of total recycling pool size and fractional release per AP. a, Pool size distribution. The distribution of pool sizes $\left(N_{\text {total }}\right)$ is shown in 3 experiments measuring each of 215 boutons. b, Quantal turnover/AP versus pool size. Three sequential measurements of $\Delta F$ were used to determine $F_{Q}(1 \mathrm{AP}), N_{\mathrm{Q}}$ / $\mathrm{AP}(7 \mathrm{AP})$ and $N_{\text {total }}(400 \mathrm{AP})$ in a single experiment. For the latter two experiments, dye exposures were extended to 15 and $60 \mathrm{~s}$ respectively. The scatter plot of $N_{Q} / A P$ versus the total pool size $N_{\text {total, indicates a }}$ correlation between these two quantities (correlation coefficient, 0.68) 
owing to the brevity of dye exposure relative to endocytosis delays, these number should be considered as lower limits.) If a strict, oneto-one coupling of unitary endocytic and exocytic events is assumed, our results indicate that a single AP may sometimes lead to multiple exocytic events at a single bouton ${ }^{19-21}$. It is possible, however, that boutons with higher release rates actually comprise multiple active zones, and that evoked release per active zone might be limited to a maximum of one vesicle.

In summary, we have shown that dye uptake occurs in quantal steps during evoked and spontaneous presynaptic function, that such steps have the magnitude expected for uptake into a single synaptic vesicle, and that such endocytosis can proceed in minimum steps of one single quantum. Our findings strongly support endocytic vesicle recycling models of presynaptic function in central synapses. Until now, the idea that presynaptic endocytosis occurs in steps corresponding to single vesicles has been based on ultrastructural observations. The observed temporal proximity of singlequantum dye uptake events to single-AP stimuli also suggests that unitary exocytic and endocytic events are coupled closely in some way. Our optical method for presynaptic quantal analysis has several features that are likely to make it a very important complement to postsynaptic electrophysiological approaches: first, it is inherently insensitive to postsynaptic factors such as receptor saturation and modulation that can complicate interpretation of postsynaptic responses ${ }^{5,6,20,22}$; second, it has an inherent ability to discriminate function at individual boutons, which is difficult with electrophysiological measurements ${ }^{19,23,24}$; third, it offers high throughput because tens or hundreds of individual boutons can be analysed in parallel in a single imaging experiment; and finally, it is both convenient and amenable to long-term experimentation because no recording pipette needs to be introduced. We expect that these features will prove highly useful in the ongoing analysis of presynaptic function and plasticity.

Note added in proof: In a recent report ${ }^{25}$ another example of multimodal FM1-43 distribution is indicated in the inset of Fig. 2.

\section{Methods}

Most experimental details have been described ${ }^{18}$. Briefly, hippocampal CA1CA3 regions were dissected from 4-day-old Sprague-Dawley rats, dissociated and plated onto Matrigel-coated coverslips and cultured for 3-5 weeks before use. Coverslips were mounted in a laminar-flow microscope chamber and continuously superfused with a saline solution consisting of $119 \mathrm{mM} \mathrm{NaCl}$, $2.5 \mathrm{mM} \mathrm{KCl}_{2} 2 \mathrm{mM} \mathrm{CaCl}_{2}, 2 \mathrm{mM} \mathrm{MgCl}, 25 \mathrm{mM}$ HEPES (buffered to $\mathrm{pH} 7.4$ ), $30 \mathrm{mM}$ glucose. Synaptic transmission was blocked by $10 \mu \mathrm{M}$ 6-cyano-7nitroquinoxaline-2,3-dione (CNQX; Research Biochemicals) $50 \mu \mathrm{M} \mathrm{D,L-2-}$ amino-5-phosphonovaleric acid (AP-5; Research Biochemicals) to prevent recurrent firing of unstimulated APs. Low- $\mathrm{Ca}^{2+}$ saline consisted of $0.5 \mathrm{mM}$ $\mathrm{CaCl}_{2}$ and $3.5 \mathrm{mM} \mathrm{MgCl}$, but otherwise identical components. The dyeloading solution contained $15 \mu$ M FM 1-43 (Molecular Probes). For experiments to resolve individual quanta, dye exposures were kept brief (typically $7 \mathrm{~s}$ ) to minimize background membrane staining. FM1-43 does not affect frequencies or amplitudes of spontaneous excitatory post-synaptic currents (EPSCs) measured in separate electrophysiological experiments (A. Bouron and H.R., unpublished results), indicating that the dye does not alter presynaptic function. APs were stimulated by passing 1-ms current pulses between electrodes placed at opposite ends of the perfusion chamber. In control experiments in which cells were stained with Fluo-3 AM, calcium imaging showed that neurons in our hippocampal cultures fired APs in response to 1-ms current pulses at a threshold field of $\sim 4 \mathrm{~V} \mathrm{~cm}^{-1}$ in both normal and low-Ca solutions. For all FM 1-43 experiments, field strengths of $10 \mathrm{~V} \mathrm{~cm}^{-1}$ were used to ensure reliable action-potential firing. Simultaneous fluorescence and Nomarski imaging was performed with a modified Bio-Rand MRC 500 laser scanning unit coupled to a Zeiss IM-35 inverted microscope and a Nikon $40 \times$ 1.3 NA Fluor objective. Regions of culture coverslips were selected for imaging using a requirement that most boutons be in focus within a single image plane, as determined using both fluorescence and Nomarski images. If a few boutons lay outside a single focal plane, those boutons were eliminated from subsequent analyses. For each image measurement, 2 frames were averaged using a dwell time of $\sim 1.5 \mu$ s per pixel, and a sampling frequency of $\sim 150 \mathrm{~nm}$ per pixel. The attenuated beam from an argon ion laser ( 200 microwatts at $488 \mathrm{~nm}$ ) was used to slightly underfill the objective pupil. Fluorescence signals were corrected for dark current and calibrated in photoelectrons, using a factor obtained from fluctuation analysis of the photomultiplier output at different illumination intensities. Comparison of images like those in Fig. $1 \mathrm{~b}, \mathrm{c}$ with similarly acquired images of a subresolution fluorescent bead ( $50 \mathrm{~nm}$ diameter; data not shown) demonstrated that the apparent spatial extent of the synaptic fluorescence punctum is exactly as expected for a subresolution object such as a $50-\mathrm{nm}$ vesicle, given the resolution limits of this confocal microscope.

Image analysis. Fluorescence measurements were made by averaging signals over a $4 \times 4$ pixel $\left(0.36 \mu \mathrm{m}^{2}\right)$ region centred over bouton images. Boutons were selected for analysis of minimal-stimulus response based on their identification using long loading and unloading trains carried out at the end of each experiment. Boutons for which the fluorescence image shifted laterally by more than 4 pixels during the unloading $(\sim 10-20 \%$ of boutons in the field, probably resulting from motion of residual fluorescence) were also rejected. Boutons whose $\Delta F$ signal was within 1.5 standard deviations of the mean of the background noise distribution ( $\leq 26$ photoelectrons) were considered to be below threshold and were eliminated, as were any boutons whose initial fluorescence values saturated the 8-bit digitization scale. The number of boutons scored as below-threshold decreased in proportion to the magnitude of the stimulus in a given experiment. Photodetection noise and the difference signal attributable to photobleaching were estimated from analysis of image-toimage variations across serially repeated images of minimally stained boutons. Noise data from 97 boutons obtained in this way were fitted with a single gaussian with s.d. $=14.5$ photoelectrons. The photobleaching rate was $\sim 5 \%$ per image. $\Delta F$ histograms were fitted with sums of gaussian curves with equally spaced peaks and widths set to approximate the variance expected from a poisson model of photodetection noise statistics as follows:

$$
\sum_{i=1}^{4} \frac{\alpha_{i}}{\sqrt{2 \pi(i+2) \mu}} \mathrm{e}^{-\frac{1}{2}\left(\frac{(x-i)^{2}}{(i+2) \mu}\right.}
$$

where $\mu$ is the integral spacing of the means, $\alpha_{i}$ is the amplitude of each gaussian, and $x$ is the position along the $\Delta F$ axis. The gaussian approximation of the underlying poisson process is valid as $\mu>10$. The standard deviation of each gaussian, $i$, was set to $\sqrt{(i+2) \mu}$, the expected dispersion arising from the two sequential measurements: image A consists of fluorescence from $i$ stained vesicles, each contributing $\mu$ photoelectrons, plus a residual equal to $\sim \mu$ photoelectrons; image B consists only of the residual of $\mu$ photoelectrons. As the histograms consist of a difference measurements of $\mathrm{A}-\mathrm{B}$, the total variance is the sum of variances from each measurement $((i \times \mu+\mu)+\mu)$. The unconstrained variables in this case are the individual amplitudes for each peak $\left(\alpha_{i}\right)$, and the centre of the first peak, $\mu$. In contrast to classical quantal analysis ${ }^{1}$, in which measurements on a given synapse are repeated serially, the peak amplitudes cannot be derived from poisson statistics as we are performing only a single measurement on each of an array of synapses of unknown statistical homogeneity.

Received 26 February; accepted 13 May 1997.

1. Katz, B. The Release of Neural Transmitter Substances (Liverpool University Press, 1969).

2. Redman, S. Quantal analysis of synaptic potentials in neurons of the central nervous system. Physiol. Rev. 70, 165-198 (1990).

3. Korn, H. \& Faber, D. S. Quantal analysis and synaptic efficacy in the CNS. Trends Neurosci. 14, 439445 (1991).

4. Kullmann, D. M. Quantal analysis using maximum entropy noise deconvolution. J. Neurosci. Meth. 44, 47-57 (1992).

5. Edwards, F. A. Anatomy and electrophysiology of fast central synapses lead to a structural model for long-term potentiation. Physiol. Rev. 75, 759-787 (1995).

6. Stevens, C. F. Quantal release of neurotransmitter and long-term potentiation. Neuron 10 (suppl.), 55-63 (1993).

7. Betz, W. J. \& Beswick, G. S. Optical analysis of synaptic vesicle recycling at the frog neuromuscular junction. Science 255, 200-203 (1992).

8. Betz, W. J., Mao, F. \& Bewick, G. S. Activity-dependent staining and destaining of living motor nerve terminals. J. Neurosci. 12, 363-375 (1992).

9. Ryan, T. A. et al. The kinetics of synaptic vesicle recycling measured at single presynaptic boutons. Neuron 11, 713-724 (1993).

10. Reuter, H. Measurements of exocytosis from presynaptic nerve terminals reveal heterogeneous inhibition by $\mathrm{Ca}^{2+}$ channel blockers. Neuron 14, 773-779 (1995).

11. Betzig, E. \& Chichester, R. J. Single molecules observed by near-field scanning optical microscopy. Science 262, 1422-1425 (1993).

12. Funatsu, T., Harada, Y., Tokunada, M., Saito, K. \& Yanagida, T. Imaging of single fluorescent molecules and individual ATP turnovers by single myosin molecules in aqueous solution. Nature 374, 555-559 (1995). 
13. Mertz, J., Xu, C. \& Webb, W. W. Single-molecule detection by two-photon-excited fluorescence. Optics Lett. 20, 2532-2534 (1995)

14. Ha, T. et al. Probing the interaction between two single molecules: fluorescence resonance energy transfer between a single donor and a single acceptor. Proc. Natl Acad. Sci. USA 93, 6264-6268 (1996).

15. Ryan, T. A., Smith, S. J. \& Reuter, H. The timing of synaptic vesicle endocytosis. Proc. Natl Acad. Sci. USA 93, 5567-5571 (1996)

16. Harris, K. M. \& Stevens, J. K. Dendritic spines of CA 1 pyramidal cells in the rat hippocampus: serial electron microscopy with reference to their biophysical characteristics. J. Neurosci. 9, 2982-2997 (1989)

17. Malgaroli, A. LTP expression: hanging like a yo-yo? Semin. Cell Biol. 5, 231-241 (1994).

18. Ryan, T. A. \& Smith, S. J. Vesicle pool mobilization during AP firing at hippocampal synapses. Neuron 14, 983-989 (1995).

19. Stevens, C. F. \& Wang, Y. Facilitation and depression at single central synapses. Neuron 14, 795-802 (1995).

20. Tong, G. \& Jahr, C. E. Multivesicular release from excitatory synapses of cultured hippocampal neurons. Neuron 12, 51-59 (1994)

21. Debanne, D., Guerineau, N. C., Gahwiler, B. H. \& Thompson, S. M. Paired-pulse facilitation and depression at unitary synapses in rat hippocampus: quantal fluctuation affects subsequent release. J. Physiol. 491, 163-176 (1996).

22. Edwards, F. A. LTP is a long-term problem. Nature 350, 271-272 (1991).

23. Bekkers, J. M. \& Stevens, C. F. NMDA and non-NMDA receptors are colocalized at individua excitatory synapses in cultured rat hippocampus. Nature 341, 230-233 (1989).

24. Liu, G. \& Tsien, R. W. Properties of synaptic transmission at single hippocampal synaptic boutons. Nature 476, 404-408 (1995)

25. Murthy, V. N., Sejnowski, T. J. \& Stevens, C. F. Heterogeneous release properties of visualized individual hippocampal synapses. Neuron 18, 599-612 (1997).

Acknowledgements. This work was supported by grants from the NIH (S.J.S.) and the Swiss Nationa Science Foundation (H.R.). We thank W. Hopf for neuronal cultures used in some experiments.

Correspondence and requests for materials should be addressed to T.A.R. at Cornell (e-mail: taryan @mail.med.cornell.edu).

\section{Cloning and characterization of} a mammalian proton-coupled metal-ion transporter

\section{Hiromi Gunshin, Bryan Mackenzie*, Urs V. Berger, Yoshimi Gunshin ${ }^{\star}$, Michael F. Romero $\dagger$, Walter F. Boron $\dagger$, Stephan Nussberger*, John L. Gollan $\neq$ \& Matthias A. Hediger $\star^{\$}$}

* Renal Division, Department of Medicine, Brigham \& Women's Hospital and Harvard Medical School, 77 Avenue Louis Pasteur, Boston, Massachusetts 02115, USA

$\dagger$ Department of Cellular \& Molecular Physiology, Yale University, New Haven, Connecticut 06510, USA

¥ Gastroenterology Division, Department of Medicine, Brigham \& Women’s Hospital, and $\$$ Department of Biological Chemistry \& Molecular Pharmacology, Harvard Medical School, Boston, Massachusetts 02115, USA

Metal ions are essential cofactors for a wealth of biological processes, including oxidative phosphorylation, gene regulation and free-radical homeostasis. Failure to maintain appropriate levels of metal ions in humans is a feature of hereditary haemochromatosis ${ }^{1}$, disorders of metal-ion deficiency, and certain neurodegenerative diseases ${ }^{2}$. Despite their pivotal physiological roles, however, there is no molecular information on how metal ions are actively absorbed by mammalian cells. We have now identified a new metal-ion transporter in the rat, DCT1, which has an unusually broad substrate range that includes $\mathrm{Fe}^{2+}$, $\mathrm{Zn}^{2+}, \mathrm{Mn}^{2+}, \mathrm{Co}^{2+}, \mathrm{Cd}^{2+}, \mathrm{Cu}^{2+}, \mathrm{Ni}^{2+}$ and $\mathrm{Pb}^{2+}$. DCT1 mediates active transport that is proton-coupled and depends on the cell membrane potential. It is a 561-amino-acid protein with 12 putative membrane-spanning domains and is ubiquitously expressed, most notably in the proximal duodenum. DCT1 is upregulated by dietary iron deficiency, and may represent a key mediator of intestinal iron absorption. DCT1 is a member of the 'naturalresistance-associated macrophage protein' (Nramp) family ${ }^{3-5}$ and thus its properties provide insight into how these proteins confer resistance to pathogens.

In mammals, iron is thought to be transported into cells by transferrin-receptor-mediated endocytosis. However, since the available apo-transferrin in the intestinal lumen (that arising from biliary excretion) is insufficient to account fully for dietary iron absorption $^{6}$, it is likely that other non-receptor-mediated uptake systems exist in the intestine. Dietary iron comprises two forms, haem and non-haem iron. The bulk of intestinal non-haem iron is absorbed in the first portion of the duodenum, in which the acidic environment promotes solubilization of iron rendered in its 2+ reduced state by ferrireductase $\mathrm{e}^{7,8}$ and ascorbate ${ }^{9,10}$. How $\mathrm{Fe}^{2+}$ is subsequently absorbed however is poorly understood. The divalent cation transporters identified so far include the mono- or oligospecific transporters derived from plants and yeast ${ }^{11}$. In addition, mammalian divalent cation export systems have been described for zinc $^{12}$ and copper ${ }^{13}$, but a mammalian uptake system has not yet been identified.

We report the expression cloning, tissue distribution and initial characterization of a divalent-cation transporter, DCT1. By screening for iron uptake activity in oocytes, we isolated the DCT1 complementary DNA from a cDNA library prepared using duodenal messenger RNA from rats fed on a low-iron diet $(\mathrm{Fe}(-) \mathrm{D})$. Xenopus oocytes injected with $\mathrm{Fe}(-) \mathrm{D}$ mRNA showed a 7-fold increase in ${ }^{55} \mathrm{Fe}$ uptake compared with water-injected (control) oocytes (Fig. 1). Following size-fractionation, maximal ${ }^{55} \mathrm{Fe}$ uptake activity was induced by a 4.0-4.5-kilobase (kb) mRNA fraction, from which we isolated a single cDNA (DCT1) which stimulated ${ }^{55} \mathrm{Fe}^{2+}$ uptake 200-fold (Fig. 1). The 4,409-base-pair (bp) DCT1 cDNA encodes a 561-amino-acid protein with 12 putative membrane-spanning domains (Fig. 2), predicted glycosylation sites in the fourth extracellular loop, and a consensus transport motif in the fourth intracellular loop ${ }^{3-5}$. In bacteria, this motif is believed to participate in the interaction with ATP-coupling subunits; however its function is unknown and it is distinct from the nucleotidebinding fold of $\mathrm{ABC}$ transporters.

High-stringency northern-blot analysis of DCT1 transcripts (Fig. 3a) revealed prominent bands of $4.5 \mathrm{~kb}$ from proximal intestine, kidney, thymus and brain, and fainter bands in testis, liver, colon, heart, spleen, skeletal muscle, lung, bone marrow and stomach. By loading an equal amount of each mRNA, we found that DCT1 was expressed at a much higher level in proximal intestine than in

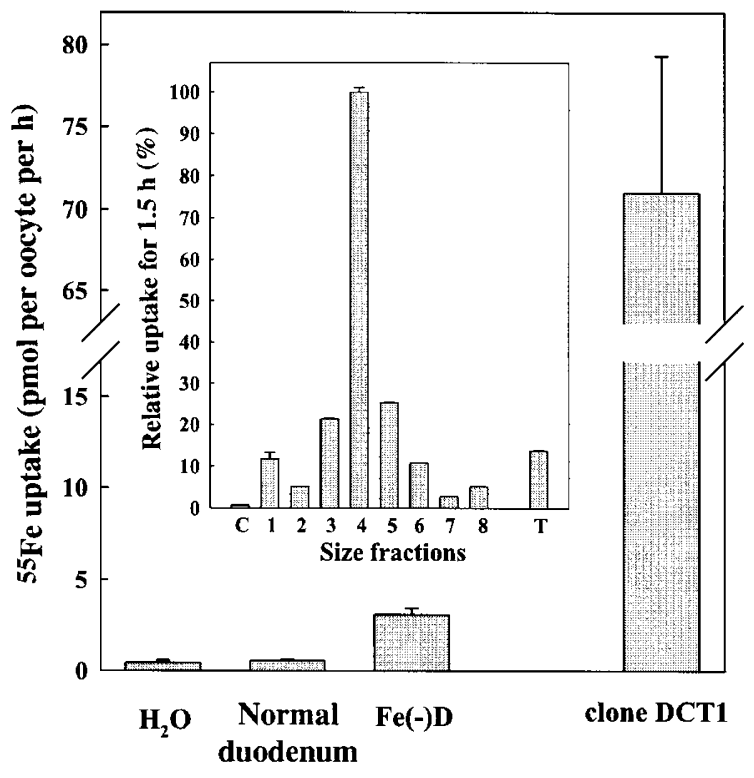

Figure 1 Uptake of $10 \mu \mathrm{M}{ }^{55} \mathrm{Fe}$ in Xenopus oocytes injected with poly $(\mathrm{A})^{+}$RNA from normal or iron-deficient $(\mathrm{Fe}(-) \mathrm{D})$ rat duodenum or with RNA synthesized from DCT1 cDNA. Data are mean \pm s.e.m. from 6-10 oocytes. Inset, relative ${ }^{55} \mathrm{Fe}$ uptake in oocytes injected with size-fractionated poly $(A)^{+} \mathrm{Fe}(-) \mathrm{D}$ RNA. C, Control water-injected oocytes; 1, 2.0-3.0 kb poly $(A)^{+} \mathrm{RNA} ; 2,2.5-3.5 \mathrm{~kb} ; 3,3.3-4.0 \mathrm{~kb} ; 4$, 3.8-4.5 kb; 5, 4.4-5.7 kb; 6, 4.5-6.0 kb; 7, 4.8-6.4 kb; 8, 5.5-7.0 kb; and T, unfractionated poly(A) ${ }^{+} \mathrm{Fe}(-) \mathrm{D}$ RNA. 